\title{
Le renouveau islamique dans l'expérience politique du Kollectif de Bondy (2000-2001)
}

The Islamic Revival in the Political Experience of the Kollectif of Bondy (2000-2001)

El renacimiento islámico en la experiencia política de Kollectif de Bondy (2000-2001)

\section{Alexandre Piettre}

\section{(Q) OpenEdition}

\section{Journals}

Édition électronique

URL : https://journals.openedition.org/remi/6662

DOI : 10.4000/remi.6662

ISSN : $1777-5418$

Éditeur

Université de Poitiers

Édition imprimée

Date de publication : 1 décembre 2013

Pagination : 111-132

ISBN : 979-10-90426-10-8

ISSN : 0765-0752

Référence électronique

Alexandre Piettre, «Le renouveau islamique dans l'expérience politique du Kollectif de Bondy (2000-2001) », Revue européenne des migrations internationales [En ligne], vol. 29 - n4 | 2013, mis en ligne le 01 décembre 2016, consulté le 16 avril 2022. URL : http://journals.openedition.org/remi/6662 ; DOI : https://doi.org/10.4000/remi.6662 


\section{Le renouveau islamique dans l'expérience politique du Kollectif de Bondy (2000-2001)}

\section{Alexandre Piettre ${ }^{1}$}

\section{Introduction. Une sociologie de l'espace public hantée par la communauté}

La sociologie de l'espace public, en puisant à la fois dans les philosophies de l'espace public, la sociologie urbaine de l'École de Chicago et la microsociologie d'Erving Goffman, a proposé une approche particulièrement féconde pour comprendre comment l'espace public urbain se constituait à travers des jeux d'interactions, en deçà de toute institution d'un espace public politique, si bien que le paradigme interactionniste est aujourd'hui épistémologiquement lié à toute sociologie se donnant l'espace public pour objet. Ce qui se joue dans l'espace public en termes de " mitoyenneté ", de côtoiement, de scène d'apparition, précède et excède en effet ce qui le définit en tant que lieu d'exercice de la citoyenneté (Joseph, 2007 : 216 ; Cefaï, 2007). Cependant, la question de l'adéquation de cette sociologie à une approche de l'espace public, du point de vue de la théorie politique, a été aussitôt ouverte (Quéré, 1992). Et de ce point de vue, on peut se demander si certains de ses attendus ne sont pas liés à une " hantise d'un devenir communautaire de la ville " (Stavo-Debauge, $2003: 347$ ), elle-même tributaire d'une conception de l'espace public présupposant qu'il doit produire un " accord " rationnel entre les citoyens (Joseph, $1998: 13$; Habermas, 1992).

Chez Isaac Joseph, cette hantise se cristallise dans une conception de l'espace public fondée sur le principe d'accessibilité. En effet, ce qui se donne d'abord comme une définition élémentaire de l'espace public, c'est-à-dire un espace où tout le monde peut aller, paraît s'ériger au fil de son œuvre comme une norme qui définit les frontières de l'espace public, au lieu d'être la source même de sa continuelle redéfinition et réinvention. En effet, en constituant l'accessibilité comme horizon positif de la " la fantasmagorie d'une urbanité démocratique " qui ferait tenir ensemble " communauté " et " citoyenneté " (1998:6-7), Isaac Joseph paraît occulter le fait que c'est en se donnant des frontières normatives que l'espace public "réellement existant " s'établit, notamment en qualifiant de

1 Docteur en sociologie du CSPRP, Université Paris 7 ; ISSRC, Université de Lausanne, Anthropole 5089, CH-1015 Lausanne, Suisse ; a_piettre@yahoo.fr 
" privé " ce qui ne doit pas y apparaître (Fraser, 2005 : 137). Ainsi, les recherches concernant l'espace public "virtuel " montrent bien que c'est par rapport à ce que les systèmes d'acteurs et de représentations donnent ou non à voir qu'un espace public se configure, puisqu'un espace entièrement ouvert comme le web confine l'immense majorité de ce qui s'y trouve, à travers les référencements, à une complète invisibilité (Cardon, 2010).

En outre, en faisant référence au concept de fantasmagorie, Isaac Joseph semble oublier qu'aux yeux de ce dernier, "I'humanité sera en proie à une angoisse mythique tant que la fantasmagorie y trouvera une place " (Benjamin, 1989 : 48), et que l'enjeu est donc de rompre le charme de la fantasmagorie pour que l'utopie qu'elle recèle puisse advenir. Si bien qu'on peut se demander dans quelle mesure le principe d'accessibilité, quand il est érigé en norme définissant I'espace public, ne fonctionne pas plutôt comme un mythe qui trace des frontières $d$ 'autant plus étanches à cet espace qu'il permet de les dénier. Ainsi Isaac Joseph identifie-t-il sur un mode fantasmagorique le passant, en tant qu'il passe inaperçu dans l'espace public, à la figure de l'étranger, du " migrant " (Joseph, 2007), en I'assimilant à l'opérateur même du " droit à l'indifférence " (ibid. : 217) qu'est censé garantir l'espace public démocratique. Or, le migrant ou l'étranger n'est-il pas précisément celui pour qui passer inaperçu dans l'espace public est une gageure, comme par exemple les sans-papiers, les " jeunes de cité " et les femmes voilées, l'éprouvent quotidiennement?

Certes, cette réalité n'a pas échappé à Isaac Joseph, mais elle est encastrée, au nom d'une "écologie urbaine " se réclamant de l'École de Chicago, dans une " naturalisation du regard sociologique que propose le modèle écologique [...] sur l'étrangéité comme rapport des populations urbaines entre elles ", si bien que "le sociologue s'intéresse moins à l'immigré dans sa spécificité et dans sa différence culturelle [...] qu'à ce qui fait de lui un tout venant et au travail de socialisation de celui qui n'est pas chez lui " (Joseph, $2007: 215-216)$. Aussi n'accorde-t-il à l'étranger qu'un "droit à l'indifférence " qui lui est pourtant toujours dénié, même lorsque l'immigré " parle comme tout le monde " (ibid. : 219). Or, n'est-ce pas lorsque l'étranger revendique son étrangeté et crée un " contrepublic subalterne " (Fraser, $2005:$ 126) pour affirmer sa différence face au " tout venant ", qu'il se donne la capacité de pouvoir " [négocier] le sens commun de I'intervalle qui les sépare " (Joseph, $2007: 217$ ) et une chance de pouvoir, un jour, passer inaperçu dans l'espace public ?

En outre, on peut remarquer qu'avec l'approche de la dramaturgie de la vie urbaine d'Erving Goffman, Isaac Joseph focalise son attention sur le " rapport spectacle/assistance [...] qui intéresse le scénographe " pour analyser l'espace public, mais fait peu de cas du "rapport scène/coulisse " (1998: 23-24) dans la mesure où dans les coulisses cesserait l'urbanité, c'est-à-dire les interactions en public et l'attention de tous les instants qu'elles nécessitent (Goffman, 1973). Or, dans son enquête auprès de militants associatifs américains, Nina Eliasoph a " constaté exactement le contraire [...] : invariablement, ce qui était proféré à voix haute était moins nuancé, moins marqué par l'esprit public, moins soucieux de l'intérêt général et plus clairement égoïste que ce qui était chuchoté " (2010: 14). Par ailleurs, James C. Scott (2008), qui a longuement étudié les comportements politiques de groupes dominés au sein de systèmes politiques autoritaires, met en évidence l'existence d'un texte caché des résistances " subal- 
ternes ", circulant en coulisse, qui prend le contrepied du texte public porté par les groupes dominants, et qui se publicise de façon fragmentaire et disruptive dans certaines circonstances exceptionnelles. Menées dans des terrains que tout devrait opposer, ces deux recherches conduisent à relativiser les frontières normatives tracées en théorie politique entre communauté et publicité, y compris dans la théorie critique ${ }^{2}$, ces frontières étant elles-mêmes tributaires de l'opposition société/communauté héritée des pères fondateurs de la sociologie moderne (Esposito, 2000). Ce problème se pose dans toute son acuité s'agissant du rôle du renouveau islamique dans les mobilisations collectives des quartiers populaires, avéré notamment pour les émeutes de 2005 (Piettre, 2006 et 2013 ; Mohammed, 2007 ; Kepel, 2012). En effet, la question est alors moins de savoir ce qui oppose scène et coulisse, public et commun, que de comprendre à quelles conditions et dans quelles circonstances ce qui se dit en coulisse devient public, ce qui fait communauté au regard d'une économie morale déterminée (Fassin, 2009) fait irruption dans l'espace public.

Or, la perspective de l'impolitique ouverte en philosophie morale et politique par Roberto Esposito peut être un point d'appui précieux pour comprendre ce qui se joue en coulisse du point de vue du politique. Cela ne se limite pas à la circulation cachée d'un discours contestataire, à ce que tente de cerner James C. Scott avec la notion d'infrapolitique. En effet, l'impolitique désigne plus largement tout ce qui échappe au politique, au-delà même de l'idée, juste au demeurant, que tout peut le devenir en se publicisant. Pour autant, il se distingue de l'apolitique comme de l'antipolitique dans la mesure où il ne renvoie à aucune réalité extérieure au politique, "soustraite au conflit du pouvoir " (Esposito, 1996 : 63). Si bien que l'impolitique n'est que " le politique observé à partir de son confin, de sa limite, de sa blessure qui l'interrompt dans son accomplissement même " (ibid. : 62). Saisie de la sorte comme limite et condition du politique, la perspective de l'impolitique récuse tout autant la confusion antipolitique entre éthique et politique, la " valorisation du politique " - Esposito désignant cette confusion comme caractéristique de toute "théologie politique " (ibid.) -, que l'approche apolitique qui consiste à les opposer radicalement sur un plan ontologique afin de disqualifier le politique d'un point de vue moral. Car ces deux positions se rejoignent in fine pour dénier à l'action politique la pluralité qu'elle manifeste en l'assimilant à l'expression d'une souveraineté homogénéisante (Arendt, 1972 : 214). Dès lors, la perspective de l'impolitique peut conférer à la subjectivation éthique orientée vers la question de la vérité, en tant qu'elle procède d'un souci de soi, de la construction de soi dans un rapport à un code moral déterminé (Foucault, 1984) ${ }^{3}$, une portée politique dans les " situations-frontières " (Arendt, $1981: 217$; Esposito, $2005: 88$ ), quand en deçà de toute action stratégique ou

\footnotetext{
2 On retrouve cet antagonisme ontologiquement construit entre communauté et publicité chez la plupart des auteurs qui, contrairement à Habermas, envisagent le public comme production d'un dissensus. Par exemple Étienne Tassin (1991), Nancy Fraser (2005: 132), ou encore Jacques Rancière (2004) qui, analysant la controverse autour du voile comme révélation d'un racisme des élites, n'envisage pas pour autant le port du voile comme producteur d'une conflictualité (Göle, 2013).

3 Elle se distingue en ce sens de la subjectivation politique orientée vers la question de l'égalité (Rancière, 1995), dans la mesure où celle-ci procède de l'exposition et de la révélation de soi dans I'espace public (Arendt, 1961). Si cela suppose un écart, cela n'implique pas nécessairement un antagonisme entre éthique et politique, mais plutôt des modalités d'articulation de l'une à l'autre dans un processus de subjectivation.
} 
organisée, les normes qu'elle cherche à habiter (Mahmood, 2009) et les référents qu'elle fait circuler innervent des interactions discordantes dans un espace public aux frontières normatives déterminées (Göle, 2005 et 2013).

La perspective de l'impolitique nous ouvre donc des horizons encore peu explorés pour appréhender le renouveau islamique comme support de politicité $^{4}$ : non seulement à l'écart d'une approche en termes d'idéologie politique, qui réduirait tout engagement public du lieu de l'islam à de l'islamisme, mais aussi pour le voir à l'œuvre en deçà de tout engagement public au nom de I'islam. À l'heure où celui-ci semble de plus en plus assumé par les acteurs sur le terrain, faire retour sur une mobilisation politique, dont le renouveau islamique a constitué le support sans que les acteurs ne s'en réclament publiquement, peut mettre en évidence la façon dont la circulation des normes et des référents islamiques opèrent comme support de politicité avant même toute intention des acteurs manifestée en ce sens. Et permettre d'élucider, ainsi, ce qui revient au religieux ${ }^{5}$ dans la configuration d'un " contre-public subalterne " (Fraser, 2005 : 126) des quartiers populaires.

C'est à l'émergence d'un tel contre-public auquel j'ai été confronté lors d'une enquête par observation participante menée durant dix-huit mois à partir de février 2000 auprès du Kollectif de Bondy, en Seine-Saint-Denis. Mouvement politique initié par des acteurs associatifs du grand quartier d'habitat social de Bondy-Nord" 6 , "enfants illégitimes " (Sayad, 2006) de l'immigration maghrébine, il a animé des luttes autour d'enjeux de gestion urbaine et a été à l'origine d'une liste " citoyenne ", la liste Rebondir, mise sur pied grâce à une alliance avec des dissidents locaux du Parti socialiste et des Écologistes indépendants. Présentée aux élections municipales de mars 2001 contre la majorité sortante dirigée par les socialistes, cette liste s'est inscrite dans la mouvance des Motivé-e-s de Toulouse, qui avaient bénéficié d'une importante exposition médiatique à la faveur de I'implication des musiciens du groupe "Zebda ». La liste Rebondir a obtenu près de $13 \%$ des voix au premier tour ${ }^{7}$, soit un des meilleurs résultats obtenus par une liste du label Motivé-e-s. Surtout, celle de Bondy est la seule à

\footnotetext{
4 Concept que I'on doit à Denis Merklen (2009) qui préfère parler de politicité que de "rapport au politique " des classes populaires, évitant ainsi de suggérer un rapport d'extériorité trompeur de ces dernières au champ politique. Ce concept me paraît d'une grande fécondité heuristique, à condition de ne pas désigner avec lui une prédisposition ontologique au politique des classes populaires, ce à quoi Denis Merklen lui-même tend à le réduire " au risque du populisme " (2009: 295). Mais il me semble opératoire au regard de la perspective de l'impolitique, dans la mesure où il permet de récuser toute opposition ontologique entre le politique et l'éthique située qui en constitue le support.

5 II s'agit de prendre au sérieux les acteurs quand ils disent « agir en tant que musulmans ", y compris lorsque leurs revendications ne se limitent pas au registre identitaire, et donc d'interroger la distinction, tributaire de l'expérience religieuse occidentale, entre religion et politique en tant que sphères autonomes et séparées d'activité et de savoirs (Id Yassine, 2012).
}

6 Le quartier concentrait près de $30 \%$ des 46800 habitants que comptait la ville au recensement de 1999.

7 La liste Rebondir avait face à elle la liste d'union de la gauche conduite par le PS $(45,85 \%)$, et celles du Front national $(17,02 \%)$, de Démocratie libérale $(15,2 \%)$ et du RPR $(9,03 \%)$. On peut évaluer ce résultat qui a permis à Rebondir d'imposer une quadrangulaire au second tour, en le comparant à ceux des élections de 1995 et de 2008 où la liste conduite par le PS avait pour seule concurrence à gauche une liste écologiste indépendante aux alentours de $9 \%$. 
avoir obtenu ses meilleurs résultats dans les quartiers populaires ${ }^{8}$, tandis que toutes les autres, et singulièrement celle de Toulouse $^{9}$, les ont récoltés dans les quartiers centraux anciens en voie de gentrification. L'implantation forte des acteurs est certainement une des clefs d'analyse, mais les autres listes ont aussi compté en leur sein des acteurs de ces quartiers.

En revanche, il semble que seule la liste Rebondir ait bénéficié de la cristallisation d'un mouvement social local qui a eu le renouveau islamique pour support, bien que cette islamité ne fut publiquement perceptible que de façon très fragmentaire. Aussi n'est-ce que par une longue fréquentation des coulisses de la campagne, jusqu'au sein de la mosquée, qu'elle pouvait être exhumée. $C^{\prime}$ est donc en analysant le système d'acteurs du Kollectif, les actions collectives qu'il a animées durant la campagne électorale, et enfin la façon dont le renouveau islamique l'a innervé, que j'éluciderai la signification politique de cette expérience et sa valeur paradigmatique pour rendre compte de la transformation de l'espace des luttes des quartiers populaires au cours des années 2000.

\section{Entre militants des cités et nouveaux acteurs islamiques, I'ambivalence structurelle du Kollectif}

\section{Sur la scène et dans la salle, les initiateurs du Kollectif}

La première réunion du Kollectif s'est tenue le 2 février 2000. Elle avait été annoncée par un tract quasi-anonyme, rédigé à la forme passive et ne portant label d'aucune organisation, appelant à mettre en œuvre la démocratie participative dans la perspective des élections locales. L'identité des quatre personnes qui ont initié le mouvement $\mathrm{n}^{\prime}$ a été révélée publiquement en ces termes et dans cet ordre qu'au cours de la troisième réunion : "Slimane, Djamel, Marwan, Leila $^{10} "$. Alors que le premier et le troisième présidaient les réunions, en présentant la démarche et en distribuant la parole depuis l'estrade, le deuxième et la quatrième relançaient les débats depuis la salle.

Des deux initiateurs les plus en vue du mouvement, Marwan et Slimane, seul Marwan s'est retrouvé par la suite en première ligne de la joute électorale, comme candidat aux élections cantonales et numéro deux de la liste Rebondir Slimane n'y apparaissant qu'en dixième position. Tous deux de nationalité française, enfants de Bondy-Nord, âgés alors de trente-quatre ans, ils avaient suivi leur scolarité ensemble, et poursuivi des études longues (Marwan en histoire, Slimane en école de médecine), tout en étant animateurs socioculturels au début des années 1990. Depuis Marwan était devenu enseignant spécialisé auprès d'enfants handicapés, alors que Slimane était sur le point d'achever

\footnotetext{
$817,07 \%$ sur les trois bureaux du nord de la ville, entre 13,11\% et $14,52 \%$ sur les cinq bureaux avoisinant trois petits quartiers d'habitat social du centre-ville et $13,62 \%$ dans le bureau du quartier populaire de la pointe sud-est de la commune ; $14,45 \%$ des voix pour le candidat du mouvement sur le canton nord-ouest, dont 20,08\% sur les trois bureaux du grand quartier nord.

9 Voir Le Monde du 13 mars 2001.

10 Pour des raisons d'anonymat, les prénoms des acteurs du mouvement ont été modifiés.
} 
son cursus. Ils étaient des piliers de l'association Tous ensemble, I'association des jeunes de Bondy Nord, Marwan en étant le président. Le partage des rôles durant la campagne électorale s'est donc effectué dans la continuité de celui qui prévalait pour leur engagement associatif. Pourtant, c'est Slimane qui généralement introduisait et présidait les réunions publiques en portant le discours public du Kollectif sur la démocratie participative, alors que Marwan, beaucoup plus disert en coulisse que sur la scène, se positionnait avant tout comme défenseur des intérêts des habitants du quartier.

Quant à Djamel et Leila, leur position était plus fragile dans l'espace social des quartiers populaires de Bondy. Originaire du quartier de Lattre de Tassigny, un quartier d'habitat social situé dans le centre-ville, Djamel avait " fait " la Marche pour l'égalité de 1983. Algérien de nationalité, donc non-éligible, il approchait les quarante ans, mais restait célibataire. Lui aussi avait fait des études supérieures, en sociologie. Il était devenu une figure du mouvement social de l'immigration et des quartiers, après avoir fondé sur Bondy l'association SOS ça bouge et le festival Y a d'la banlieue dans l'air en 1988 avec le soutien de la municipalité. Puis il s'est consacré à partir des années 1990 à un travail de documentariste en Algérie" ${ }^{11}$ et a vu ses espoirs de " carrière " dans l'action culturelle locale déçus après l'élection du nouveau maire socialiste Gilbert Roger en 1995. Animé d'une véritable rancœur à son encontre, il vivait depuis dans une réelle précarité chez ses parents à Villeparisis. Enfin Leila était dotée d'un solide capital culturel et social : titulaire d'un diplôme de niveau II, âgée de trente-cinq ans, d'origine algérienne, elle avait entamé sa carrière militante en République centrafricaine aux côtés d'un opposant à la dictature, et était employée comme chargée de mission sur les politiques sociales auprès du Conseil Général. Mais elle vivait seule avec un enfant, et habitait depuis peu la ville : elle avait emménagé en 1997 dans un F2 d'un nouveau programme de logements sociaux, en périphérie immédiate des cités de Bondy Nord. Le quartier ne constituait donc pas pour elle un milieu d'inter-connaissances. Elle avait rencontré Marwan et Slimane à travers des réseaux de sociabilité extérieurs à Bondy, et n'a finalement été placée qu'en vingt-neuvième position sur la liste Rebondir.

La présence de cette jeune femme issue de l'immigration mais étrangère au quartier éclaire les conditions d'émergence du Kollectif. En effet, lors des premières réunions, Slimane et Marwan ne se sont pas réclamés de la légitimité de Tous ensemble, ni n'ont invoqué leur parcours militant sur la ville. Ils ne voulaient donc pas que leur entreprise politique puisse être assimilée à leur action associative. On peut mettre en regard leur attitude avec celle de Djamel qui ne cessait de rappeler son action passée, trahissant l'affaiblissement de sa position dans I'espace social. Ainsi, pour passer à I'action politique, Slimane et Marwan ont au préalable effacé leurs traces ${ }^{12}$. D'où aussi l'importance, capitale, de la présence de Leila parmi les initiateurs, elle qui n'avait aucun titre à faire valoir comme militante locale. Ainsi peut-on dire avec Walter Benjamin, que I'en-

11 Aujourd'hui décédé, il a réalisé deux documentaires qui ont été diffusés à la télévision : avec Michel Vuillermet, Algérie, mémoire du raï, France, Les Films du Village, 2001, $52 \mathrm{mn}$, et Cheb Hasni, je vis encore, Algérie, Huit \& Plus Productions, 2008, 52 mn.

12 Motif récurrent dans les articles de Walter Benjamin des années 1930, notamment dans Le Caractère destructeur, Brèves ombres et Expérience et pauvreté (2000a: $330-332,353,370)$, les traces désignent ces rebuts d'expérience dont il convient de se dépouiller pour ne pas rester sous I'emprise de "I'habitude " et pouvoir agir. 
treprise politique du Kollectif ne pouvait que procéder d'une certaine destruction de l'aura des "œuvres " accomplies, le festival, les voyages organisés, ou encore les chantiers-jeunes de réfection des halls qui avaient donné lieu à la réalisation d'un petit film documentaire ${ }^{13}$. C'était la condition, en tout cas, pour ouvrir l'espace du mouvement social à de nouveaux acteurs et à une temporalité autre que celle de la Politique de la ville.

\section{Entre arène et coulisses, des " jeunes de cité " aux nouveaux acteurs islamiques}

À travers les rapides portraits que je vais maintenant dresser de quelques hérauts plus discrets du mouvement, je vais tenter de donner un bref aperçu des différents réseaux dont ils constituent la pointe émergée, ainsi que des modalités de leurs connexions. Ainsi, un point qui a d'emblée caractérisé le Kollectif des premières réunions (février-avril 2000), c'est la présence d'une bonne dizaine de " garçons arabes " âgés de vingt-cinq à trente-cinq ans, faiblement dotés en capital social et culturel, mais qui gravitaient auparavant autour de Tous ensemble. S'ils furent très présents au début dans la salle, conférant aux premières réunions du mouvement leur caractère d'exutoire du texte caché circulant dans les halls, ils se tiendront souvent à une certaine distance de la campagne électorale de Rebondir. Mais ils formeront le gros des troupes que I'on retrouvera plus loin dans le groupe Unis-cités, à la veille des élections. Parmi eux, Mehdi et Atman qui, avec une formation initiale de niveau IV en poche, avaient trouvé dans les organisations du quartier des supports précieux dans leur trajectoire, notamment à la faveur du dispositif emploi-jeunes, grâce auquel ils avaient été embauchés par la mairie à la maison de quartier Daniel Balavoine pour Mehdi, et dans I'association Y'a d'la banlieue dans I'air pour Atman. Ils habitaient tous deux Bondy Nord chez leurs parents, Mehdi étant le cadet d'une fratrie très réputée, voire crainte, sur le quartier. En effet, ses deux grands frères dirigeaient l'association de "bienfaisance ", La Voie droite, d'obédience salafi. Pour autant, il n'était pas lui-même engagé dans une démarche pieuse, et rien ne pouvait laisser soupçonner chez lui pareille affiliation.

Mais une telle connexion aux réseaux islamiques est loin d'être isolée : au-delà de leur propre pratique religieuse éventuelle (respect des interdits alimentaires et observance du jeûne le plus souvent), elle concerne de près ou de loin tous les acteurs engagés dans le mouvement, comme par exemple Marwan, dont la sœur aînée a pris le voile. Mentionnons, parmi les anciens militants actifs de Tous ensemble, les cas d'Adnan et d'Akim qui avaient adhéré au Parti socialiste et qui l'ont quitté pour rejoindre le Kollectif. Sans que cela ne soit visible extérieurement (ils restaient glabres), ils étaient eux-mêmes croyants et pratiquants (prières quotidiennes, ramadan, fréquentation épisodique de la mosquée le vendredi). En outre, ils étaient liés en grande amitié - et le sont

13 Voir L'œuvre d'art à l'époque de sa reproductibilité technique (Benjamin, 2000b : 269-316). Pour Walter Benjamin, trace et aura sont les pendants I'une de l'autre, et c'est donc l'effacement de la première qui permet la destruction de la seconde : " Avec la trace, nous nous emparons de la chose ; avec l'aura, c'est elle qui se rend maîtresse de nous " (ibid., 1989 : 464). 
toujours aujourd'hui ${ }^{14}$ - avec les deux acteurs islamiques que nous allons présenter ci-après.

C'est en tout cas du côté des " nouveaux acteurs islamiques " (Göle, 2002) que le Kollectif a trouvé des ressources nouvelles. Le mouvement a en effet été investi par quelques musulmans pieux, i.e. dont l'engagement religieux est ostensible (modes de présentation de soi distinctifs) et de notoriété publique (fréquentation quotidienne de la mosquée). Les plus notables furent Étienne le converti, présent depuis le départ, et Djibril arrivé pour préparer la campagne électorale en septembre 2000, assistant même à des réunions en petit comité au domicile de la tête de la liste Rebondir. Ces deux individus ont chacun un profil très singulier qui rend compte de leur engagement assez exceptionnel dans le Kollectif et la campagne de Rebondir, par rapport aux autres musulmans pieux qui s'en tinrent généralement à distance.

Étienne le converti avait une trentaine d'années et restait célibataire. Il était arrivé cinq ans auparavant sur le quartier pour occuper le poste d'intendant du collège de Bondy Nord. II était très bien doté en capital social et culturel. Venu à I'islam à la suite d'une adolescence passée en Algérie, il manifestait le zèle religieux des nouveaux convertis, laissant le plus possible pousser sa barbe, et sortant de chez lui en qamis tant qu'il pouvait. Son double statut d'agent de l'État et de musulman pieux faisait de lui un point à double entrée : il cultivait à la fois des relations amicales avec la future tête de liste de Rebondir, le directeur de la SEGPA ${ }^{15}$, et avec certains militants de Tous ensemble, notamment avec Adnan et Akim qu'il avait connus par l'intermédiaire d'un surveillant du collège. En outre, il avait l'estime des plus jeunes à la mosquée, lui permettant d'intégrer ce qu'il appelle à dix ans de distance, avec une pointe de fierté mêlée d'ironie, sa "bande ", une dizaine de jeunes de seize à trente ans qui se disaient proches des Frères musulmans, attentifs aux écrits et aux propos du cheikh Al Qaradâwî et de I'intellectuel Tariq Ramadan $^{16}$. Ce point le singularisait aux yeux de ses "frères ", dans la mesure où les quatre autres Français convertis qui fréquentaient la mosquée s'inscrivaient dans l'association des salafis.

Djibril avait quant à lui trente-trois ans, était marié et avait deux filles. D'origine algérienne, il se distinguait par un solide capital culturel, avec une formation de niveau II et une culture religieuse très étoffée. II était travailleur indépendant dans le domaine informatique, ayant semble-t-il monté une affaire avec un de ses frères, ce qui lui permettait de cultiver sa formation éthique sans

$14 \mathrm{~J}$ 'ai entretenu des contacts réguliers avec les acteurs après l'enquête, notamment avec Adan et Étienne. Avec ce dernier, j'ai réalisé un entretien le 15 septembre 2010 revenant sur toute cette expérience, à une époque où sa piété s'était beaucoup relâchée. Toutes les citations de lui ci-après proviennent de cet entretien.

15 Les Sections d'enseignement général et professionnel adapté sont destinées aux élèves présentant des difficultés d'apprentissage ne leur permettant pas de suivre le cursus " normal " du collège unique.

16 Célèbre religieux qatariote d'origine égyptienne, animant l'émission La shariah et la vie sur Al Jazira, le cheikh Al Qaradâwî dirige depuis 1997 le Conseil européen de la recherche et de la fatwa afin d'élaborer un " fiqh de minorité ", une jurisprudence islamique d'exception adaptée au contexte européen. Tariq Ramadan s'en distingue par ses prises de position en faveur de la construction d'une communauté musulmane européenne qui ne se considère plus comme une minorité tolérée ou invitée (Ramadan, 1999), et qui doit pouvoir faire évoluer I'ensemble de la jurisprudence islamique (ibid., 2003). 
les entraves que les musulmans pieux rencontrent la plupart du temps dans un contexte laïc. Aussi était-il réputé pour sa piété irréprochable : toujours habillé à la " mode " salafi, au minimum avec une chemise blanche très longue et un pantacourt dégageant les chevilles comme était réputé faire le Prophète, faisant régulièrement la tournée des petites mosquées salafistes de la région (Étienne l'accompagnant à l'occasion), il ne portait pas pour autant de jugement sur les pratiques moins "sunna " des autres fidèles fréquentant la mosquée. Mais il était précisément l'un des rares auquel le noyau dur des salafis du quartier, qui rassemblait une vingtaine de personnes au sein de La Voie droite, daignait parler, cette " distinction " particulière s'expliquant aussi par le fait qu'un de ses frères cadets, Karim, faisait partie de ce groupe fonctionnant sur un mode très sectaire.

La présence de nouveaux acteurs islamiques au sein du Kollectif converge jusqu'à un certain point avec des observations déjà faites à l'époque, à propos de jeunes inscrits dans la mouvance $i k h w a n^{17}$. Toutefois, ce qui était remarquable dans le contexte bondynois et $\mathrm{n}^{\prime}$ a été que rarement observé depuis à ma connaissance - alors que rien ne permet de penser qu'il s'agit d'un cas isolé -, c'est à la fois la proximité de l'ensemble des obédiences du renouveau islamique (ikhwan et salafis en l'occurrence) avec le Kollectif, et le fait que la plupart de ses acteurs sont dans le même temps restés en retrait de la mobilisation politique. Ce n'est donc pas seulement, ni même essentiellement, en tant que pourvoyeurs de militants que le renouveau islamique a été présent au mouvement social.

\section{Du Kollectif à Rebondir: mouvement social, alliance et divorce avec une frange de la gauche locale}

\section{La cristallisation d'un mouvement social autour d'enjeux de gestion urbaine}

À Bondy, la mobilisation initiée par le Kollectif a pu déboucher sur la présentation d'une liste aux élections municipales dans la mesure où il a animé un mouvement social qui s'est déployé durant toute la durée du processus électoral. Celui-ci s'est cristallisé autour d'enjeux de gestion urbaine au sens large, depuis le ramassage des ordures jusqu'aux chantiers de réhabilitation, en passant par les horaires d'ouverture des maisons de quartier.

En effet, en encadrant l'implication des habitants dans le conflit ${ }^{18}$ et en obligeant le maire à s'impliquer dans les négociations entre les ouvriers et leur employeur, le Kollectif a permis les 7 et 8 juin 2000 la satisfaction des revendications des éboueurs en grève sur la commune : maintien des employés sur la ville de Bondy et du treizième mois, paiement des jours de grève. Cette grève

\footnotetext{
17 Dénomination arabe des Frères musulmans. L'engagement citoyen de ces jeunes, vécu comme une modalité nodale de leur engagement religieux, était un phénomène émergent déjà bien documenté, l'exemple le plus emblématique à cet égard étant celui de I'Union des Jeunes Musulmans à Lyon (Cesari, 1998).

18 Intervention pour empêcher des intérimaires de nettoyer le marché de Bondy Nord et d'ainsi casser la grève, et organisation d'une marche des habitants du quartier jusqu'à la mairie.
} 
avait été entamée une semaine auparavant, suite au transfert de la délégation de service public d'une société privée de ramassage des ordures à une autre, empêchant les marchés de se tenir, notamment le marché tri-hebdomadaire à Bondy Nord infesté de rats. II est remarquable que la force de ce mouvement social soit corrélée au refus des grévistes, eux-mêmes habitants le quartier de Bondy Nord pour la plupart, de travailler ailleurs que dans la ville, la proximité entre habitat et emploi étant vécue comme aussi précieuse que le niveau du salaire. C'est à la suite de cette victoire qu'une alliance fut nouée durant l'été avec des dissidents du Parti socialiste proches du courant de la gauche socialiste, puis à l'automne avec la mouvance écologiste indépendante locale, pour présenter la candidature de la liste Rebondir aux élections municipales.

Durant toute la campagne électorale, le Kollectif continua à animer des luttes " urbaines " sur Bondy Nord. Entre novembre 2000 et février 2001, il a ainsi conduit une action de longue haleine contre I'OPHLM $^{19}$ de la commune, en relayant le mécontentement des locataires à l'encontre d'une réhabilitation de 200 logements conduite sur la partie la plus ancienne du patrimoine de I'office sur Bondy Nord. Les locataires se plaignaient en effet de I'utilisation d'un " matériel à très bas prix ", ou du " carrelage [...] pas fait au-dessus de la gazinière ", des " malfaçons ", incriminant un "travail bâclé ", que le Kollectif n'a eu aucun mal à donner pour reflet de la faible considération portée aux habitants des "quartiers ". Dans le contexte de la campagne électorale, le Kollectif déborda l'amicale des locataires existante qui avait accepté le projet de réhabilitation, suite à deux rencontres avec le directeur de I'OPHLM les 17 et 31 janvier 2001. Le Kollectif obtint l'engagement du bailleur à ne pas réceptionner les travaux avant que toutes les malfaçons, dûment constatées après passage chez chaque locataire d'un contrôleur technique de l'office, fussent reprises. Et il fut invité à fonder une nouvelle amicale de locataires, de façon à pouvoir participer au conseil consultatif de gestion de l'office que la loi relative à la solidarité et au renouvellement urbains (SRU), adoptée en décembre 2000, imposait de mettre en place. Invitation honorée en ces termes par Fatima, cinquante ans, membre du Kollectif placée en cinquième position sur la liste Rebondir, et future présidente de la nouvelle amicale :

"Ça va beaucoup vous aider cette amicale, c'est très important. Je connais les gens dans l'autre amicale, mais c'est toujours les mêmes. Et les gens de notre cité, de nos origines, ne peuvent pas y aller".

Le Kollectif venait de signer là son succès plus durable ${ }^{20}$ : rien de moins qu'une victoire d'Outsiders supplantant les Établis (Elias et Scotson, 1997). En tout cas, l'une et l'autre de ces actions ont entraîné la légitimation publique du mouvement. Toutefois, cette légitimation passait par le filtre du texte public en vigueur relatif à la démocratie participative, qui faisait écran aux ressorts profonds de la mobilisation.

19 Office Public d'Habitations à Loyer Modéré.

20 L'Association pour la Défense des Locataires de I'OPHLM de Bondy, dont les statuts ont été déposés en préfecture le 19 juin 2001, existe toujours aujourd'hui. 


\section{Des événements révélateurs d'un conflit de subjectivation avec les acteurs de la gauche traditionnelle au sein de la liste Rebondir}

Au cours de la campagne électorale, les leaders du Kollectif ont envoyé plusieurs signaux aux musulmans pieux, de façon tantôt cachée, tantôt publique. Ainsi, Marwan et Slimane ont laissé discrètement les clés du local de Tous ensemble à La Voie droite. Cela était de "notoriété publique " dans les sites sociaux subalternes, en premier lieu à la mosquée, mais n'a jamais été évoqué publiquement durant toute la campagne électorale. Simultanément, à la veille du Ramadan, les leaders du Kollectif ont obtenu de la municipalité qu'au lieu d'une arrière-salle dans le très vétuste centre social Giono, la nouvelle maison de quartier Balavoine puisse être ouverte le soir, et en ont fait un élément de propagande inscrit à l'ordre du jour de la première réunion publique de Rebondir qui s'est tenue à Bondy Nord le $1^{\text {er }}$ décembre 2000, en lui donnant la même importance que la lutte contre la réhabilitation.

Par ailleurs, les occurrences de la publicisation de l'islamité des acteurs tendirent à se faire plus nombreuses à I'approche du scrutin. En effet, I'alliance s'était nouée sur un " deal " concernant l'ordre d'apparition sur la liste entre un dissident du Parti socialiste comme tête de liste, François S., et Marwan en second de liste. À cet arrangement s'est joint ensuite François D., écologiste indépendant, en troisième position. Or, l'absence de procédures pour légitimer ces choix auprès des acteurs du Kollectif, outre qu'elle vidait de sa substance la démocratie participative que la liste prétendait incarner, eut surtout pour effet de dénier au Kollectif une fonction hégémonique dans la campagne, la tête de liste cherchant à lui substituer un " comité de soutien des 2001 " et à instituer I'assemblée des colistiers comme seule instance de délibération sur la conduite de la campagne. Les résistances des acteurs du Kollectif prirent alors des formes contradictoires, allant de l'esquive à la contestation ouverte, en passant par la ruse. L'émergence d'un petit mouvement "dissident ", "Unis-cités ", jouant sur la graphie pour évoquer à la fois l'univers des cités et l'unicité divine en islam ( Tawhid) ainsi que I'a explicité son dirigeant, Kader, un ancien " jeune de cité " de Tous ensemble et des premières réunions du Kollectif, fut l'alerte la plus sérieuse. II tenta de se réapproprier le capital politique du mouvement en fustigeant ceux qui auraient voulu le récupérer (ceux " qu'on ne connaît pas " dans la liste), affichant l'intention de voter pour Marwan aux cantonales, mais pas pour la liste Rebondir, appelant à rallier le maire sortant Gilbert Roger et organisant même avec lui une réunion publique.

Ces soubresauts ont plus révélé qu'entravé la dynamique née avec le Kollectif: le succès de la liste Rebondir au premier comme au second tour (avec encore près de $12 \%$ des voix) en témoigne. Et plus encore le succès personnel de Marwan comme candidat aux cantonales sur les bureaux de vote des cités de Bondy Nord, creusant à chaque fois des écarts de près de trois points avec la liste Rebondir. Entre lui et François S., il y avait la tonalité des professions de foi, très différentes. Alors que celle de François S. se fiait "au Bondynois " et aux futurs conseils d'habitants pour tous les sujets de la vie municipale, celle de Marwan consistait à rappeler les avancées concrètes obtenues avec le Kollectif en faveur des éboueurs et des habitants, puis en faveur des locataires de I'OPHLM. En outre, elle plaçait sa candidature sous le double signe de la société 
assurantielle, avec une citation de Léon Blum en exergue (" "Toute société qui prétend assurer aux hommes la liberté, doit commencer par leur garantir l'existence" "), et de l'éthique islamique introduite ainsi (" et parce que nous croyons encore à la politique, il nous appartient d'en rétablir l'éthique "), avec une proposition qui aurait paru tout à fait incongrue ou déplacée pour un tenant de la gauche progressiste, car relative aux normes islamiques de pudeur dans la vie quotidienne et à la prescription coranique de "commander le bien " (amr bi-l maarouf) et de " pourchasser le mal " (hisba) :

" Je m'engage à [...] demander la création d'une unité mobile départementale de nettoyage dont la mission sera de lutter contre l'affichage sauvage d'encarts publicitaires à caractère pornographique $n^{21}$.

C'est donc un véritable conflit de subjectivation (Bayart, $2004: 217$ ), resté largement dans l'ombre, qui opposait les acteurs du Kollectif à leurs alliés à gauche de l'échiquier politique local, et qui est à l'origine de la disjonction finale de Rebondir. S'il minait profondément leurs relations et leurs interactions, il ne reposait cependant sur aucun clivage politique qui fût énoncé. François S., élu le 18 mars en même temps que Marwan, démissionna de son mandat sans explication le 23 mai. Et dès le 26 juin, François D., siégeant au conseil municipal à la faveur de cette démission, rendit publique la séparation entre les deux élus avec cette communication écrite :

"Comme d'autres, je crois toujours que la diversité de nos origines culturelles et sociales est une richesse mais comme tout "programme commun", ce n'est pas "un long fleuve tranquille" [...]. Aujourd'hui, je crois indispensable que chacune des deux composantes de notre liste puisse reprendre leur travail séparément en gardant comme boussole les objectifs contenus dans notre "programme commun" et sur lequel nous avons été élus ".

Ainsi, le naufrage final de l'expérience, malgré l'épreuve de la sanction électorale passée avec succès, tient au fait que ce conflit de subjectivation n'a pas été énoncé publiquement par les acteurs, sinon de façon trop fragmentaire ou elliptique à l'approche et au lendemain des élections. Toutefois, il est bien à la fois à l'origine du succès électoral et à l'origine de la division entre les " deux composantes " de la liste Rebondir, de I'interaction discordante entre deux espaces-temps, celui qui borne l'espace public établi autour de repères bien identifiés depuis la Révolution, et celui qui s'y est inscrit de façon disruptive et " anachronique " en y introduisant des normes religieuses dont les acteurs font remonter la généalogie au VIle siècle (Göle, 2013).

Du côté des acteurs du Kollectif, l'absence d'énonciation publique, sinon fragmentaire, de ce conflit de subjectivation, est liée à sa dimension impolitique. En effet, les opinions politiques des acteurs, la proximité avec tel ou tel courant théologico-politique de l'islam, pèsent ici beaucoup moins que l'action de quelques individus au sein et à la périphérie du mouvement comme " entrepreneurs de morale ", au travers de l'ascèse et des techniques du corps qu'ils s'imposent. Ce faisant, ils déjouent la construction sociale de la déviance

21 Texte de la propagande officielle pour le premier tour de la campagne électorale du candidat soutenu par Rebondir aux élections du conseiller général du canton nord-ouest de Bondy. 
qui suppose l'existence d'une contre-culture entièrement tributaire de sa stigmatisation, apparaissant en dernière instance comme une condition d'effectivité de la norme majoritaire (Becker, 1985). Car ce qu'implique le renouveau islamique, c'est précisément l'action d' " entrepreneurs de morale " qui, bien que minoritaires (Moscovici, 1979), ouvrent la voie à des changements sociaux majeurs en inscrivant une discontinuité entre deux systèmes éthico-normatifs dans un même espace social (Id Yassine, 2012), désamorçant ainsi cette dialectique permettant aux normes dominantes de se nourrir de leur contestation. C'est pourquoi, l'agir du Kollectif tendait à conférer à l'impolitique une portée politique, non par adhésion à une idéologie "islamiste ", mais sur un mode beaucoup plus pragmatique et performatif, par son affiliation épistémique au renouveau islamique en tant qu'il innerve un conflit de subjectivation.

\section{Le renouveau islamique comme support de politicité}

\section{La mosquée, lieu nodal d'un conflit de subjectivation}

En effet, les acteurs du renouveau islamique ont compté sans doute parmi les relais d'opinion les plus décisifs de Rebondir. C'est notamment le cas du réseau le plus puissant sur le quartier, celui constitué à partir du groupe organisé des salafis qui, bien que se tenant à distance du politique, soutenait discrètement le Kollectif. Mais cela n'était visible que lorsqu'ils venaient prendre au marché des piles de tracts qu'ils mettaient sur leur étal d'articles et de livres pieux.

Ainsi, durant toute la campagne électorale, les discussions religieuses et politiques, au sein des "bandes " de jeunes fidèles ont agité la mosquée de façon beaucoup plus intense qu'à l'ordinaire, accusant un clivage entre les générations. En effet, l'initiative du Kollectif de présenter une liste a été très mal reçue par le président de l'association gérant la mosquée : pour lui, c'était là faire preuve d'ingratitude à l'égard du maire qui venait de délivrer un permis de construire pour une mosquée ${ }^{22}$. À l'occasion de l'Aïd-el-Fitr, le 27 décembre 2000 , juste après le sermon en arabe, il avait pris l'initiative de lancer un appel en français et en arabe à voter pour le maire sortant. Cela avait beaucoup choqué la " bande " des ikhwan, moins sur le principe même qu'il puisse soutenir le maire sortant, que sur le lieu (la mosquée), le moment (le jour de l'Aïd), et la manière (I'emploi du français pour l'appel à voter mais pas pour le sermon) qu'il avait choisis pour le communiquer.

En effet, le clivage était plus éthique que politique : celui-ci se cristallisait à la mosquée sur des points particuliers de fiqh al-ibadat, c'est-à-dire de jurisprudence concernant les obligations religieuses et rituelles, ou de fiqh al-mu'amalat

\footnotetext{
22 Ce fut la première "vraie " mosquée construite en Seine-Saint-Denis. D'une superficie de $570 \mathrm{~m} 2$, située à proximité des cités de Bondy Nord, elle a été inaugurée en mars 2005, et entièrement financée par les fidèles, la municipalité n'ayant même pas eu à exercer un droit de préemption ou à céder un terrain. Jusque-là, la mosquée était située dans le grand ensemble, dans un local au pied d'une grande barre d'immeuble de I'OPHLM, en rez-de-chaussée mais en contrebas des entrées, à un niveau où il n'y a pas de logement - d'où sa caractérisation à l'époque comme " cave " par les fidèles. On peut mesurer le chemin parcouru depuis avec la première pierre posée en 2013 d'un centre culturel musulman, distinct de la mosquée.
} 
qui concerne l'ensemble de la sociabilité, des interactions sociales, depuis les règles à observer lorsqu'on se lève, se lave, boit et mange jusqu'aux impératifs à respecter en matière de transactions financières. Par exemple, durant la campagne électorale, un problème de fiqh al-ibadat concernant la prière a opposé principalement les salafis de La Voie droite aux anciens et à l'imam malékite traditionnel. Les fidèles se rendant à la mosquée de façon quotidienne y allaient principalement pour la prière du soir (Maghrib) et y restaient éventuellement jusqu'à celle de la nuit (Isha). Si l'on peut faire plus de cinq prières par jour, une règle sanctionnée par un hadith " authentique ${ }^{23}$ veut cependant qu'on ne fasse pas de prière supplémentaire entre la prière de l'après-midi $(A s r)$ et celle du soir. Mais une autre règle veut que lorsqu'on y entre, on fasse toujours deux rakat (deux cycles de prière) de salutation à la mosquée. Or, traditionnellement, les anciens ne font pas ces deux rakat au moment d'entrer à la mosquée pour Maghrib, au contraire des plus jeunes, qu'ils s'agissent des salafis ou des ikhwan. II s'agit là d'un véritable marqueur entre les générations, entre musulmans traditionnels d'origine maghrébine se conformant généralement à la jurisprudence malékite, et acteurs du renouveau islamique n'hésitant pas à explorer l'ensemble des écoles jurisprudentielles pour déterminer leur conduite (Mahmood, 2009 : 131). Pour les salafis, ne pas faire ces deux rakat de salutation à la mosquée était vraiment indigne. "Sur le fond ", comme le disait Étienne, les "frères " pensaient qu'ils avaient raison, mais ils estimaient qu'en la circonstance la tolérance prévalait :

"II y a un hadith pour les deux rakat, donc normalement la question est résolue. Mais pour les anciens, c'est la tradition, ils ne se posent pas de question. Et les vieux, eux, ils ne disaient rien aux jeunes s'ils ne faisaient pas comme eux. Donc, vraiment, ça ne pouvait poser problème qu'aux salafis".

En effet, les salafis faisaient de ces questions de fiqh al-ibadat un enjeu de pouvoir pour étendre leur emprise au sein de la communauté, exigeant par exemple en janvier 2001 la démission de l'imam à propos d'une pratique soufie des anciens - les lectures collectives du Coran entre les deux prières du soir à la mosquée - qu'ils considéraient comme une " innovation blâmable " (bida'a), Étienne comparant à ce propos celui qui dirigeait le groupe salafiste à un "émir du GIA ". Pour autant, j'y insiste, il ne s'agissait pas pour la " bande " des ikhwan d'un point mineur ou secondaire. Le fiqh al-ibadat et le figh al-mu'amalat étaient au cœur de leurs discussions informelles quand ils se retrouvaient le soir à la mosquée, et la quête de leurs lectures. Point d'émir cependant parmi eux, mais seulement Sofiane qui avait un peu plus de "science " que les autres, capable notamment de lire les corpus de Hadith à la source, en arabe, et avec leur " chaîne de transmission " permettant d'évaluer leur " degré d'authenticité ". Parmi les points techniques qui, entre eux, prêtaient à discussion et à exploration des Hadiths et des quatre écoles jurisprudentielles, il y avait par exemple la question de savoir jusqu'à quel point on peut tailler sa barbe en se conformant toujours au hadith qui enjoint aux musulmans de " tailler leur moustache et de laisser aller leur barbe ".

23 Dont la chaîne de transmission, des compagnons du Prophète qui l'ont rapporté oralement jusqu'au moment où il a été consigné, est considérée comme " sûre ". 
Certes, cette " bande " de "frères " ne s'interdisait pas, entre eux, de parler d'autre chose que de fiqh. Un de leurs sujets de conversation favoris était le football, et ils y jouaient souvent ensemble, même en qamis, ce que leur reprochaient particulièrement les salafis : " pour eux, on était avec le diable parce qu'on parlait de foot ". Et ils parlaient fréquemment de politique, notamment de politique internationale et de la Palestine, pour laquelle ils ne manquaient aucune manifestation. Mais aussi beaucoup de politique locale à l'approche des élections municipales, leurs avis pouvant diverger fortement concernant le Kollectif. Ainsi, sans y être hostile, Sofiane était le plus pessimiste quant à la capacité d'une telle mobilisation à peser sur la situation, considérant que l'observation du fiqh al-ibadat et du fiqh al-mu'amalat était plus à même de le faire. De sorte qu'il pourrait sembler plus proche des salafis que deTariq Ramadan sur la question.

\section{La capacité d'agir conférée par le renouveau islamique}

Aussi ne faut-il pas chercher de distinction nette entre les deux tendances du côté de leur posture vis-à-vis du politique, toutes deux liées à une subjectivation éthique orientée vers la question de la vérité. Si l'engagement d'Étienne au sein du Kollectif n'est a priori pas très étonnant, dans la mesure où il se disait proche de Tariq Ramadan, celui de Djibril semble au premier abord plus surprenant du fait de sa proximité avec les salafis, ceux-ci enjoignant aux musulmans de rester à l'écart de l'espace public en dehors de la " terre d'Islam ". Mais si I'on se place maintenant du côté des problématisations à l'origine de leurs " modes de subjectivation ", de leur formation éthique, de I' " ascétique " ou des "pratiques de soi " qu'ils mettent en œuvre pour l'assurer (Foucault, $1984: 40$ ), il apparaît qu'il n'y a pas de frontière nette, comme s'il s'agissait de doctrines ou d'idéologies politiques opposées, entre les deux principales tendances de la salafiya.

Ainsi, Étienne et Djibril lisaient et suivaient tous deux La Voie du musulman (Minhaj el Moslim), une sélection de Hadiths qui codifient un mode de vie islamique dans ses plus infimes détails (comment manger, dormir, se laver, etc.), opérée par un cheikh saoudien salafi originaire d'Algérie (Djaber El Djazaïri, 2006). Cet ouvrage, qu'on trouve dans toutes les librairies musulmanes, est parmi I'un des livres pieux les plus vendus en France après Le Jardin des vertueux (Riyâd as-Sâlihîn), qui date du Moyen Âge et qui constitue toujours aujourd'hui la synthèse la plus accessible et la plus respectée de la Sunna à destination du fidèle non-savant (An-Nawawi, 2007). Voici comment Étienne dit qu'il lisait et utilisait La Voie du Musulman :

" Je l'utilisais surtout sur les conditions de validité des ablutions, de la prière, sur des trucs vraiment techniques. Dans le Minhaj el Moslim, il n'y a aucune spiritualité, il n'y a que de la technique. Ce n'était pas un bouquin qu'on lisait comme ça, c'était un manuel, c'était ardu parce que parfois les techniques, quand il n'y a pas de dimension spirituelle... Enfin, ça dépend, par exemple, faire attention quand tu te chausses, commencer par le côté droit, pour moi ça a une dimension spirituelle. Je le fais toujours aujourd'hui, mais je ne fais plus attention, c'est devenu une habitude, et là il n'y a plus de dimension spirituelle. En fait, l'important, c'est de faire attention, c'est ça, c'est de faire attention. C'est du dhikr, ça veut dire rappel, souvenir ("souvenez-vous de moi, je me souviendrai de vous" [Coran, S. 2, v. 152]), et c'est ce qui fait qu'il y a une dimension spirituelle dans

l'acte". 
Véritable manuel de technologie de soi, ce qu'Étienne cherchait donc dans cet ouvrage, c'était avant tout des techniques de soi permettant une permanente "remémoration" (Foucault, 2001 : 1618). Ainsi, I'influence religieuse et culturelle des salafis est sans commune mesure avec celle de leur idéologie politique qui assimile l'Oumma à un corps politique, comme du temps du califat. En effet, aux yeux d'Étienne qui adhère à la démarche politique d'un intellectuel commeTariq Ramadan et rejette sans ambiguïté celle des salafis, ceux-ci offrent cependant un " mieux-disant " du point de vue du mode de vie islamique, dans leur recherche systématique d'imitation des " pieux ancêtres " (i.e. les compagnons du prophète, les salafs) et leur lutte contre toute bida'a. Cela convenait en tout cas à sa quête de pratiques performatives de transformation de soi, de performances rituelles à même de le former comme sujet pieux.

Ainsi, si I'on s'en tient au registre de Foucault qui paraît ici le plus adéquat, les différences entre ikhwan et salafis du côté de "l'ascétique ", c'est-à-dire des techniques de soi, ne sont pas significatives, dans la mesure où elles participent d'une même " détermination de la substance éthique " entrant dans la constitution du rapport à soi : elles permettent "le strict respect des interdits et des obligations " tout comme "le combat acharné qu'on mène contre [les désirs] ", ce qui suppose dans les deux cas une "vigilance " et une "lutte " constante, et les mêmes " mouvements contradictoires de l'âme " (Foucault, 1984 : 38). Certes, les différences peuvent être significatives en termes de problématisation de la conduite éthique, c'est-à-dire de " mode d'assujettissement " (de la " façon dont l'individu établit son rapport à [la] règle et se reconnaît comme lié à I'obligation de la mettre en œuvre ") et de " téléologie du sujet moral ", en tant qu'une action n'est pas morale seulement parce qu'elle se conforme à un code, mais aussi du fait qu'elle participe de la constitution du sujet moral (ibid. : 38-39). Reste que les uns et les autres se retrouvent du même côté d'un conflit de subjectivation qui les oppose au reste de la société "libérale ", car " la conception que l'on a du sujet requiert et présuppose une forme spécifique d'engagement politique " (Mahmood, 2009 : 58). En l'occurrence, elle implique que cet engagement se déploie sur un mode plus pragmatique qu'idéologique, en restant tributaire de la réflexivité que les acteurs du renouveau islamique exercent à travers des discours, des procédures et des techniques qui "visent avant tout l'amélioration de leurs capacités intellectuelles et émotionnelles afin de se rapprocher du modèle exemplaire du soi pieux " que constitue le Prophète (Mahmood, 2009 : 56).

On touche là à l'essentiel de ce qui se joue dans le renouveau islamique (salafiya, au sens générique de retour aux fondements de l'islam) : celui-ci est intelligible dans une perspective impolitique qui ne fait pas de tri a priori entre ses différentes tendances, dans la mesure où son premier enjeu est la transformation de soi et qu'il n'est pas orienté vers une visée de changement social dans une perspective progressiste (Mahmood, 2009). Mais c'est précisément à ce point que le renouveau islamique acquiert une dimension politique, dans la mesure où ces pratiques performatives de transformation de soi innervent une visibilité disruptive de l'islam dans l'espace public qui, avant même toute intention des acteurs en ce sens, pose d'emblée la question des frontières de I'espace public, c'est-à-dire la question de savoir si l'acteur islamique peut ou non y apparaître (Göle, 2005 et 2013). 
Ce faisant, la politicité du renouveau islamique est irréductible à telle ou telle interprétation théologique de l'islam, à une quelconque théologie de la libération. Elle consiste essentiellement à opposer un jeu de langage à un autre, en l'occurrence un jeu de langage qui cultive une identité musulmane basée sur une interprétation ontologique de l'islam, à un autre basé sur l'idée que l'Occident des Lumières est un projet qui définit la modernité, mettant ainsi en évidence aussi bien l'hégémonie que la contingence de ce dernier (Sayyid, 2003). Et elle est fonction des interactions discordantes de ces jeux de langage, de la visibilité disruptive des performances rituelles des acteurs, qui sont en ce sens autant d'images dialectiques, de constellations saturées de "tensions" (Benjamin, 1989 : 494) au travers desquelles l'impolitique acquiert une portée politique. De ce point de vue, on s'explique mieux l'impact impolitique du salafisme sur le cours de l'expérience politique du Kollectif de Bondy, sans comparaison avec I'influence proprement antipolitique de La Voie droite, dans la mesure où il donne accès à une technologie de soi particulièrement efficiente en termes de formation éthique du sujet pieux et de visibilité publique. Au point d'apparaître comme un "réformisme générique " (Saint-Lary, 2012) largement partagé par les acteurs au travers de quelques marqueurs rituels dérivés (ici prière de salutation à la mosquée pour Maghrib, critique des rites soufis, barbe plus ou moins touffue, qamis et chevilles découvertes), et hésitant entre lecture littéraliste et pragmatique des textes sacrés, bien plus que comme une tendance sectaire clairement identifiable.

\section{Conclusion. Politicité, religiosité : les performances du renouveau islamique}

Ainsi, I'expérience politique du Kollectif a pu passer avec un certain succès l'épreuve de la sanction électorale en termes de mobilisation des quartiers populaires, dans la mesure où elle a eu un conflit de subjectivation éthique comme support de politicité. Toutefois, cette expérience est celle d'un échec, dans la mesure où cette dimension de la mobilisation est restée largement dans l'ombre du fait de l'hégémonie d'un texte public relatif à la " citoyenneté ", sanctuarisé dans le cadre de la campagne de Rebondir. Pire, son échec a été sanctionné par la réification des " origines culturelles", et non en raison d'un clivage politique, suite à la publicisation fragmentaire de ce conflit de subjectivation, participant de ce fait et malgré elle d'une dynamique de (re)construction des frontières de I'espace public établi (Salzbrunn, 2012).

Mais ce que montre tant le succès que l'échec de cette expérience, c'est que de l'impolitique au politique, il y a précisément le filtre du public, en tant qu'il soumet l'action à une " force illocutionnaire qui [...] semble configurer ici la véritable unité pragmatique de l'agent collectif " (Naishtat, 2010 : 139). Francisco Naishtat appréhende ici l'action collective au prisme de la pragmatique ${ }^{24}$, mais à l'écart du cadre institutionnalisé dont Austin (1970) et Searle (1969) avaient fait la condition de performativité des actes illocutoires, et du lieu de " sa capacité à se différencier dans le champ symbolique des forces illocutionnaires " (Naishtat,

24 Branche de la philosophie analytique du langage, la pragmatique complète la sémantique et étudie I'utilisation des signes dans des situations concrètes, notamment dans des actions collectives. 
2010 : 12). Ainsi le caractère performatif du renouveau islamique tient précisément au fait qu'il est un vecteur de force illocutionnaire capable de " générer un public " (ibid. : 198 ; Dewey, 2005) qui, en tant que tiers, soumet l'action " à des critères de félicité pragmatique ou performative, pouvant être soit résiliée, soit réalisée dans les arènes publiques " (Naishtat, 2010 : 13).

Aussi l'échec final de l'expérience du Kollectif a-t-il maintenu durablement Djibril et Étienne à l'écart de tout engagement militant (Fillieule, 2010). En effet, cet échec impliquait que malgré une certaine légitimation de l'inscription des populations issues de la migration dans l'espace social local, notamment à travers la création d'une nouvelle amicale de locataires, les acteurs du Kollectif n'étaient pas véritablement parvenus à modifier leur position subalterne dans l'espace public. Ainsi, Marwan rejoignit finalement les rangs de la majorité municipale afin de pouvoir se ménager des capacités d'action auprès des habitants de son quartier, avant de démissionner. À cet égard, il semble que les mobilisations qu'on peut aujourd'hui observer dans les quartiers populaires, en France et au-delà, témoignent d'un changement qualitatif. En effet, de façon beaucoup plus systématique et assumée qu'auparavant, mais aussi plus ambivalente (Piettre et Monnot, 2013), les acteurs revendiquent le fait d'agir en tant que musulmans et tentent d'engager à partir du référent religieux un rapport de pouvoir avec les municipalités qui les oblige à reconnaître, au-delà des mosquées où elles aimeraient les confiner, les acteurs islamiques comme des acteurs sociaux légitimes (Pingaud, 2012 ; Marzi, 2013).

Mais c'est aussi ce caractère performatif du renouveau islamique qui permet de saisir comment l'expérience du public, à travers la mobilisation du Kollectif, transforme ce qui était à l'origine un phénomène sectaire en une religiosité inédite, au travers de laquelle les référents islamiques tendent à acquérir une signification nouvelle (Foucault, 2008) par rapport à celle qui leur est conférée dans la Tradition en termes de pudeur, de réserve, de renoncement à l'intramondanité, dans la mesure même où ces normes sont investies d'une charge subversive pour forcer I'accès à I'espace public (Göle, 2013). Car le filtre du public a un effet sur les processus mêmes de formation des valeurs (Dewey, 2011) : en incluant les salafistes restant généralement en retrait du politique dans un dispositif de force illocutionnaire, l'expérience du Kollectif a neutralisé le salafisme sectaire et permis à un salafisme "générique " opérant comme support de politicité de se déployer. Ainsi, I'enjeu majeur de cette expérience ne fut rien moins qu'une nouvelle "valuation " (ibid.) des référents islamiques, ce à quoi s'emploient aujourd'hui nombre d'acteurs du renouveau islamique mobilisés à l'échelle locale et trans-locale avec une plus grande félicité illocutionnaire. 


\section{Références bibliographiques}

An-Nawawî Imam (2007) Le jardin des vertueux, Lyon, Tawhid, 798 p.

Arendt Hannah (1981) La Vie de l'esprit, vol. 1 : La pensée, Paris, PUF, 244 p.

Arendt Hannah (1972) La crise de la culture, Paris, Gallimard, 380 p.

Arendt Hannah (1961) Condition de l'homme moderne, Paris, Calmann-Lévy, $406 \mathrm{p}$.

Austin John L. (1970) Quand dire, c'est faire, Paris, Seuil, 203 p.

Bayart Jean-François (2004) Total subjectivation, in Jean-François Bayart et JeanPierre Warnier Éds., Matière à politique. Le pouvoir, les corps et les choses, Paris, Karthala, pp. 215-253.

Becker Howard S. (1985) Outsiders. Études de sociologie de la déviance, Paris, Métailié, 248 p.

Benjamin Walter (2000a) Fuvres II, Paris, Gallimard, 459 p.

Benjamin Walter (2000b) Fuvres III, Paris, Gallimard, 482 p.

Benjamin Walter (1989) Paris, Capitale du XIXe siècle. Le Livre des passages, Paris, Cerf, $974 \mathrm{p}$.

Cardon Dominique (2011) La démocratie Internet. Promesses et limites, Paris, Seuil, 102 p.

Cefaï Daniel (2007) Pourquoi se mobilise-t-on ? Les théories de l'action collective, Paris, La Découverte, 727 p.

Cesari Jocelyne (1998) Musulmans et républicains. Les jeunes, I'islam et la France, Bruxelles, Éditions Complexe, $166 \mathrm{p}$.

Dewey John (2011) La formation des valeurs, Paris, La Découverte, 237 p.

Dewey John (2005) Le public et ses problèmes, Paris, Gallimard, 337 p.

Djaber El Djazaïri Aboubakar (2006) La Voie du Musulman, Paris, Maison d'Ennour, 568 p.

Elias Norbert et Scotson John L. (1997) Logiques de l'exclusion, Paris, Fayard, $279 \mathrm{p}$.

Eliasoph Nina (2010) L'évitement du politique. Comment les Américains produisent l'apathie dans la vie quotidienne, Paris, Economica, $353 \mathrm{p}$.

Esposito Roberto (2005) Catégories de l'impolitique, Paris, Seuil, 248 p.

Esposito Roberto (2000) Communitas. Origine et destin de la communauté, Paris, PUF, $166 \mathrm{p}$.

Esposito Roberto (1996) La perspective de l'impolitique, Tumultes, 8, pp. 59-69.

Fassin Didier (2009) Les économies morales revisitées, Annales. Histoire, Sciences sociales, 64 (6), pp. 1237-1266.

Fillieule Olivier (2010) Some elements of an interactionnist approach to political disengagement, Social Movement Studies, 9 (1), pp. 1-15. 
Foucault Michel (2008) L'archéologie du savoir, Paris, Gallimard, 294 p.

Foucault Michel (2001) Dits et Écrits II, 1976-1988, Paris, Gallimard, 1735 p.

Foucault Michel (1984) Histoire de la sexualité, vol. 2 : L’Usage des plaisirs, Paris, Gallimard, 342 p.

Fraser Nancy (2005) Qu'est-ce que la justice sociale ? Reconnaissance et redistribution, Paris, La Découverte, $179 \mathrm{p}$.

Goffman Erving (1973) La mise en scène de la vie quotidienne, vol. 1 : La présentation de soi, Paris, Minuit, $251 \mathrm{p}$.

Göle Nilüfer (2013) La visibilité disruptive de I'islam dans l'espace public européen. Enjeux politiques, questions théoriques, Sens public, 15-16, [en ligne] consulté le 18 octobre 2013. URL : http://www.eurozine.com/articles/2013-09-25gole-fr.html

Göle Nilüfer (2005) Interpénétrations. L'Islam et l'Europe, Paris, Galaade Éditions, $160 \mathrm{p}$.

Göle Nilüfer (2002) Islam in Public: New Visibilities and New Imaginaries, Public Culture, 14 (1), pp. 173-190.

Habermas Jürgen (1992) L'espace public. Archéologie de la publicité comme dimension constitutive de la sphère publique bourgeoise, Paris, Payot, 324 p.

Id Yassine Rachid (2012) L'islam d'Occident ? Introduction à l'étude des musulmans des sociétés occidentales, Perpignan, Éditions Halfa, 328 p.

Joseph Isaac (2007) Le migrant comme tout venant, in Daniel Cefaï Éd., L'athlète moral et l'enquêteur modeste, Paris, Economica, pp. 211-220.

Joseph Isaac (1998) La ville sans qualités, La Tour d'Aigues, Éditions de I'Aube, 209 p.

Kepel Gilles (2012) Banlieue de la république. Société, politique et religion à Clichy-sous-Bois et Montfermeil, Paris, Gallimard, 530 p.

Mahmood Saba (2009) Politique de la piété. Le féminisme à l'épreuve du renouveau islamique, Paris, La Découverte, 312 p.

Marzi Eva (2013) Citoyennes et musulmanes. Le partage du religieux et du politique dans I'horizon de reconnaissance d'une association musulmane à Genève, in Christophe Monnot Éd., La Suisse des mosquées. Derrière le voile de I'unité musulmane, Genève, Labor et Fides, pp. 173-195.

Merklen Denis (2009) Quartiers populaires, quartiers politiques, Paris, La Dispute, $305 \mathrm{p}$.

Mohammed Marwan (2007) Les voies de la colère : "violences urbaines " ou révolte d'ordre "politique " ? L'exemple des Hautes-Noues à Villiers-sur-Marne, Socio-logos, 2, [en ligne] consulté le 11 juillet 2007. URL : http://socio-logos. revues.org/document352.html

Moscovici Serge (1979) Psychologie des minorités actives, Paris, PUF, 275 p.

Naishtat Francisco (2010) Action et langage. Des niveaux linguistiques de I'action aux forces illocutionnaires de la protestation, Paris, L'Harmattan, 260 p. 
Piettre Alexandre (2013) Islam (im)politique et quartiers (im)populaires. Retour critique sur les émeutes de 2005, L'Homme et la société, 187-188 (1), pp. 89-129.

Piettre Alexandre (2006) Les grandes " émotions " de novembre 2005. Perspectives pour un résistible nouvel échec politique à gauche, Mouvements, 43 , pp. $122-130$.

Piettre Alexandre et Monnot Christophe (2013) Entre reconnaissance et visibilité. Les luttes des acteurs islamiques en Suisse et en France, in Christophe Monnot Éd., La Suisse des mosquées. Derrière le voile de l'unité musulmane, Genève, Labor et Fides, pp. 199-231.

Pingaud Étienne (2012) Un militantisme musulman ?, Savoir/Agir, 22, pp. 61-69.

Quéré Louis (1992) L'espace public : de la théorie politique à la métathéorie sociologique, Quaderni, 18, pp. 75-92.

Ramadan Tariq (2003) Les Musulmans d'Occident et l'avenir de l'islam, Paris, Actes Sud, $390 \mathrm{p}$.

Ramadan Tariq (1999) Être Musulman Européen. Étude des sources islamiques à la lumière du contexte européen, Lyon, Tawhid, $460 \mathrm{p}$.

Rancière Jacques (2004) Le voile ou la confusion des universels, Rue Descartes, 44 (2), pp. 124-125.

Rancière Jacques (1995) La Mésentente. Politique et philosophie, Paris, Galilée, $191 \mathrm{p}$.

Saint-Lary Maud (2012) Du wahhabisme aux réformismes génériques. Renouveau islamique et brouillage des identités musulmanes à Ouagadougou, Cahiers d'études africaines, 206-207 (2), pp. 449-470.

Salzbrunn Monika (2012) Performing Gender and Religion. The veil's impact on Boundary-Making Processes in France, Women's Studies, 41 (6), pp. 682-705.

Sayad Abdelmalek (2006) L'immigration ou les paradoxes de l'altérité, vol. 2 : Les enfants illégitimes, Paris, Raisons d'agir, 207 p.

Sayyid Salman (2003) A Fundamental Fear. Eurocentrism and the Emergence of Islamism, London-New York, Zed Books Ltd, 185 p.

Scott James C. (2008) La domination et les arts de la résistance. Fragments du discours subalterne, Paris, Éditions Amsterdam, 270 p.

Searle John R. (1969) Speech Acts. An essay in the philosophy of language, Cambridge, Cambridge University Press, 214 p

Stavo-Debauge Joan (2003) L'indifférence du passant qui se meut, les ancrages du résidant qui s'émeut, in Daniel Cefaï et Dominique Pasquier Éds., Les Sens du public. Publics politiques, publics médiatiques, Paris, PUF, pp. 347-371.

Tassin Étienne (1991) Espace commun ou espace public ? L'antagonisme de la communauté et de la publicité, Hermès, 10, pp. 23-37. 


\section{Alexandre Piettre}

\section{Le renouveau islamique dans l'expérience politique du Kollectif de Bondy (2000-2001)}

À partir d'une ethnographie d'une mobilisation collective en banlieue parisienne en 2000 et 2001 qui a débouché sur la présentation d'une liste aux élections municipales grâce à une alliance avec une frange de la gauche, cet article tente d'élucider ce qui revient au religieux dans l'émergence en France d'un espace des luttes des quartiers populaires autour du référent islamique. Seule liste " citoyenne " à réaliser alors pareille performance, elle a obtenu $13 \%$ des voix et ses meilleurs scores dans les quartiers populaires en étant soutenue par des entrepreneurs de morale du renouveau islamique, avant que ses deux composantes ne se séparent après les élections en raison d'une discordance culturelle. Ainsi le succès et l'échec de la mobilisation ont moins tenu aux mots d'ordre relatifs à la " citoyenneté ", qu'à sa capacité à générer un dissensus au regard des discours, des techniques et des procédures que certains de ses acteurs mettaient en œuvre pour former leurs subjectivités pieuses.

\section{The Islamic Revival in the Political Experience of the Kollectif of Bondy (2000-2001)}

From an ethnography of a collective mobilization in the Paris suburbs in 2000 and 2001 which led to the presentation of a list in municipal elections through an alliance with a fringe of the left, this article attempts to elucidate what returns to the religious in the emergence in France of a space of suburbs struggles around the Islamic referent. Single list of "citizens neighborhoods" to then achieve such performance, she got $13 \%$ of the votes and top scores in the popular areas by being supported by moral entrepreneurs of Islamic revival, before its two components broke apart after the elections because of a cultural discordance. Thus the success and failure of mobilization were less due to the watchwords related to "citizenship", than to its ability to generate a dissent in relation to discourses, techniques and procedures that some of its stakeholders had implemented to form their pious subjectivities.

\section{El renacimiento islámico en la experiencia política de Kollectif de Bondy (2000-2001)}

A partir de una etnografía de una movilización colectiva en los suburbios de París en 2000 y 2001, que dio lugar a la presentación de una lista en las elecciones municipales a través de una alianza con un sector de la izquierda, este artículo trata de dilucidar cuál es la religión en la aparición en Francia de un espacio de luchas de los barrios en torno al referente islámico. Única lista "ciudadana» en esto caso, consiguió el $13 \%$ de los votos y sus mejores puntuaciones en los barrios pobres con el apoyo de emprendedores morales de renacimiento islámico, antes de sus dos componentes se separan después de las elecciones en debido a discordancia culturales. Y el éxito y el fracaso de la movilización eran menos debidos los lemas relacionados con la "ciudadanía" y su capacidad de generar disensión sobre discursos, técnicas y procedimientos que algunos de sus actores ponen esfuerzo para formar a sus subjetividades piadosas. 